\title{
Effects of twin-beam "squashed" light on a three-level atom
}

\author{
L.K. Thomsen and H.M. Wiseman \\ School of Science, Griffith University, Nathan 4111, Australia
}

(February 1, 2008)

\begin{abstract}
An electro-optical feedback loop can make in-loop light (squashed light) which produces a photocurrent with noise below the standard quantum limit (like squeezed light). We investigate the effect of squashed light interacting with a three-level atom in the cascade configuration and compare it to the effects produced by squeezed light and classical noise. It turns out that one master equation can be formulated for all three types of light and that this unified formalism can also be applied to the evolution of a two-level atom. We show that squashed light does not mimic all aspects of squeezed light, and in particular it does not produce the characteristic linear intensity dependence of the population of the upper-most level of the cascade three-level atom. Nevertheless, it has non-classical transient effects in the de-excitation.
\end{abstract}

42.50.Dv, 42.50.Ct, 32.80.-t

\section{INTRODUCTION}

Squeezing is a form of non-classical light which involves the reduction of noise in one quadrature below the standard quantum limit, and the increase of the noise in the conjugate quadrature [1]. However the characteristic below-shot-noise photocurrent is not limited to squeezed light. An electro-optical feedback loop can also produce an (in-loop) homodyne photocurrent with a below-shotnoise spectrum. The resultant in-loop light is called "squashed" light [2, [4. Our interest lies in understanding the non-classical effects of the interaction of squashed light with atoms.

It has recently been shown $[5]$ that illuminating a twolevel atom with squashed light causes line narrowing of one quadrature of the atom's fluorescence, an effect also produced by squeezing [6]. This effect of squeezing (or squashing) has not yet been observed [7]. However, it has been demonstrated [8] that a squeezed two-photon field exciting a three-level atom in the cascade configuration gives an excited population for the highest level with a linear as well as quadratic (classical) dependence on intensity. This effect was first predicted in Ref. [9] and the experiment has recently been analyzed by the same authors [10]. This experiment motivates our current work to see if squashing also produces effects similar to squeezing when illuminating a cascade three-level atom.

Although based on the same techniques as in [5], the development of the model for the three-level system required a complete redesign of the feedback mechanism. Non-classical excitation of a three-level atom requires non-classical correlations between two fields, i.e. a twinbeam, which couple to the two transitions in the atom. Twin-beam correlations can be produced in a double feedback system, where the fed-back photocurrents result from linear combinations of the twin-beam quadratures. These are analogous to the twin-beam squeezing correlations produced by a non-degenerate optical parametric oscillator [8]. The resultant master equation for a cascade three-level atom interacting with twin-beam squashed light can be compared to those for squeezed [9] and classical twin-beams.

The structure of the paper is as follows. Section II introduces the interaction of non-classical light with simple atomic systems. Specifically, it covers previous results of the squeezed excitation of a two-level atom, and it summarizes the production of squashed light and its interaction with a two-level atom. The techniques and concepts introduced here will be used again in later sections.

Section III presents the theory for a three-level atom coupled to a broadband squeezed field, and introduces our definition of a "twin-beam". Section IV develops the main theoretical results of this paper, which include the master equation for a three-level atom coupled to twinbeam squashed light, and the correlations of the squashed twin-beam itself.

Section V compares the non-classical nature of squashed light with squeezed light as well as classical noise. The first two parts of this section show that a general formalism can be found for all three types of light interacting with both two-level and three-level atoms. Finally, Section V also details the specific classical and nonclassical effects of squashed light. Section VI concludes.

\section{TWO-LEVEL ATOM}

The interaction of non-classical light with atoms was first studied soon after the first incontestible observation of squeezing [11]. For a recent review of atoms in squeezed light fields see Ref. [12]. It began with the prediction by Gardiner [6] that broadband squeezed light would produce an arbitrarily narrow line in the power spectrum of the fluorescence of a two-level atom. This was thought of as a "direct effect of squeezing" [6].

A recent letter [5] by one of us showed that squashed light also produces a similar line-narrowing, and so this "direct effect" is one of non-classical correlations rather than solely of squeezing. The work on squashed light spectroscopy of two-level atoms was recently extended to 
include simultaneous squeezing and squashing [3]. Here we review the two effects separately.

\section{A. Squeezed light}

The squeezed vacuum fields that are used as spectroscopic sources are actually multi-mode squeezed states. The theory of single-mode squeezing was generalized to multi-mode squeezing by Caves and Schumaker 13]. The continuum field is the limit of an infinite number of modes. For polarized light propagating in one direction, the modes are simply indexed by the mode frequency $\omega=c k$. The continuum annihilation operators $b(\omega)$ may be taken to satisfy

$$
\left[b(\omega), b^{\dagger}\left(\omega^{\prime}\right)\right]=\delta\left(\omega-\omega^{\prime}\right) .
$$

If the occupied modes are restricted to a relatively narrow bandwidth $B$ around some carrier frequency then, in the time domain, the commutation relation becomes

$$
\left[b(t), b^{\dagger}\left(t^{\prime}\right)\right]=\delta\left(t-t^{\prime}\right)
$$

where the width of the $\delta$-function is actually of the order $B^{-1}$ and $b(t)$ can now be thought of as the annihilation operator of a "localized photon". Thus $b^{\dagger}(t) b(t)$ can be interpreted as the photon flux operator. The $X$ and $Y$ quadratures of the continuum field are defined as

$$
X(t)=b(t)+b^{\dagger}(t), \quad Y(t)=i b^{\dagger}(t)-i b(t),
$$

and for free fields these obey the commutation relations

$$
\left[X(t), Y\left(t^{\prime}\right)\right]=2 i \delta\left(t-t^{\prime}\right)
$$

For fields with zero mean, as are all the fields in this paper, the $X$ quadrature spectrum is defined as

$$
\begin{aligned}
S_{X}(\omega) & =\langle\tilde{X}(\omega) X(0)\rangle_{\mathrm{ss}}, \\
& =\frac{1}{2 \pi} \int_{-\infty}^{\infty}\left\langle\tilde{X}(\omega) \tilde{X}\left(-\omega^{\prime}\right)\right\rangle_{\mathrm{ss}} d \omega^{\prime},
\end{aligned}
$$

and similarly for the $Y$ quadrature spectrum. It can then be shown that for a stationary free field

$$
S_{X}(\omega) S_{Y}(\omega) \geq 1
$$

which is known as the Heisenberg inequality [14,15]. A vacuum continuum field [16,17] is one such that for all $\omega$ and all $\theta, S_{Q_{\theta}}(\omega)=1$. Here $Q_{\theta}=X \cos \theta+Y \sin \theta$. This is called the standard quantum limit or shot-noise limit. For a squeezed continuum field we have $S_{Q_{\theta}}(\omega)<1$, for some $\omega$ and some $\theta$.

Now consider placing a two-level atom in a squeezed continuum field. Under the rotating-wave approximation the interaction Hamiltonian is

$$
H(t)=i \hbar\left[b^{\dagger}(t) \sigma(t)-\sigma^{\dagger}(t) b(t)\right]
$$

where $\sigma$ is the atomic lowering operator. Here the degree of mode matching of the input light modes to the atom's dipole radiation mode is assumed to be unity and the atomic linewidth is also set to unity. See Ref. [5] for details on mode-matching.

Assuming the squeezed light is broadband compared to the atomic linewidth, we can make the white noise approximation that the quadrature spectra are constant. For experimental generation of squeezed light (e.g. an optical parametric oscillator) photons are produced in correlated pairs with frequencies symmetrically placed around a center frequency $\omega_{0}[12$. The photon number function, $N\left(\omega_{k}\right)=\left\langle b^{\dagger}\left(\omega_{k}\right) b\left(\omega_{k}\right)\right\rangle$ and the two-photon correlation function, $M\left(\omega_{k}\right)=\left\langle b\left(\omega_{k}\right) b\left(2 \omega_{0}-\omega_{k}\right)\right\rangle$ are both constants in the broadband approximation.

In general for squeezed light $N \leq|M| \leq$ $\sqrt{N(N+1)}$, where the upper limit represents the minimum-uncertainty state, also known as the maximally squeezed state. For minimum-uncertainty squeezing with $M$ real, the light can be characterized by a single real number $L=1+2 N+2 M[\mathbb{1}]$. Thus the quadrature spectra are

$$
S_{X}(\omega)=L=1 / S_{Y}(\omega)
$$

If $M<0$ then $L<1$ and the $X$ quadrature is squeezed, i.e. $S_{X}(\omega)<1$, and correspondingly the $Y$ quadrature is stretched, i.e. $S_{Y}(\omega)>1$.

From these correlations and the interaction Hamiltonian (2.7) one can obtain the following master equation for the atom [18]:

$$
\dot{\rho}=\frac{1}{4 L} \mathcal{D}\left[(L+1) \sigma-(L-1) \sigma^{\dagger}\right] \rho,
$$

where as usual for arbitrary operators $A$ and $B$,

$$
\mathcal{D}[A] B \equiv A B A^{\dagger}-\frac{1}{2} A^{\dagger} A B-\frac{1}{2} B A^{\dagger} A
$$

From the master equation the following dynamical equations can be derived

$$
\begin{aligned}
& \operatorname{Tr}\left[\dot{\rho} \sigma_{x}\right]=-\gamma_{x} \operatorname{Tr}\left[\rho \sigma_{x}\right] \\
& \operatorname{Tr}\left[\dot{\rho} \sigma_{y}\right]=-\gamma_{y} \operatorname{Tr}\left[\rho \sigma_{y}\right] \\
& \operatorname{Tr}\left[\dot{\rho} \sigma_{z}\right]=-\gamma_{z} \operatorname{Tr}\left[\rho \sigma_{z}\right]-C
\end{aligned}
$$

where

$$
\gamma_{x}=\frac{L}{2}, \gamma_{y}=\frac{1}{2 L}, \gamma_{z}=\gamma_{x}+\gamma_{y}, C=1
$$

Here we see that, for $X$ quadrature squeezing $(L<0)$, the decay of $\sigma_{x}$ is below its natural value of $1 / 2$. In terms of the squeezed spectrum it is given by $S_{X}(\omega) / 2$. This slower decay causes a corresponding line narrowing in the power spectrum of the atom's fluorescence. 


\section{B. Squashed light}

As mentioned in the introduction, there is another approach to producing light with a sub-shot-noise photocurrent apart from squeezing: this is squashing. This involves modulating the light incident on a photodetector by using the very current from that detector, i.e. electrooptical feedback. Squashed light was first observed [19] not long after the first observation of squeezing [11].

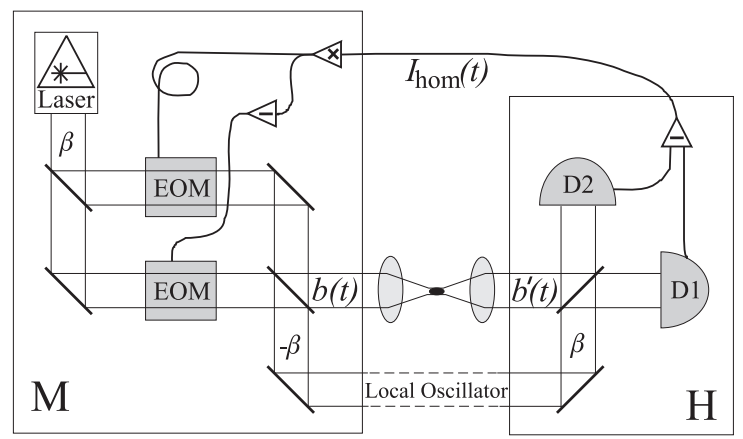

FIG. 1. Experimental configuration for squashed light incident on a two-level atom. The atom is represented by a small ellipse at the focus of $b(t)$. The detection device (region $\mathrm{H}$ ) finds the difference $I_{\text {hom }}(t)$ between the currents at the photodetectors D1 and D2. The modulation device (region M) amplifies and splits $I_{\text {hom }}(t)$ so that two signals (with opposite sign) are fed back to the two electro-optical modulators (EOM).

Consider the experimental apparatus shown in Fig. 1. We begin with the modulation region, M, which adds a modulated coherent amplitude to the input vacuum field. There are various ways of achieving this including the one pictured, which is detailed in Ref. [5]. The resultant field, $b(t)$, is incident on a two-level atom. After the atom, a homodyne measurement is performed on the field, now given by $b^{\prime}(t)$. This is done in the detection region, $\mathrm{H}$, and makes use of the local oscillator also produced in M. The homodyne photocurrent is then used to control the modulation of the coherent amplitude, and it is this feedback that ensures $b(t)$ has noise below the standard quantum limit.

The field exiting the modulator is given by

$$
b(t)=\nu(t)+\varepsilon(t),
$$

where $\nu(t)$ represents the input vacuum and $\varepsilon(t)$ is a real function of time. For a vacuum field all first and second order moments vanish except for [18]

$$
\left\langle\nu(t) \nu^{\dagger}\left(t^{\prime}\right)\right\rangle=\left[\nu(t), \nu^{\dagger}\left(t^{\prime}\right)\right]=\delta\left(t-t^{\prime}\right),
$$

and the $X$ and $Y$ quadratures have spectra, defined by (2.5), equal to unity. Thus they are the special case of (2.8) where $L=1$.

For now ignore the two-level atom. The detection device makes a homodyne measurement of the $X$ quadrature of $b^{\prime}(t)$, which in the absence of the atom is equal to $b(t)$. The homodyne photocurrent is then given by [18]

$$
I_{\mathrm{hom}}(t)=X_{\nu}(t)+2 \varepsilon(t),
$$

where $X_{\nu}(t)=\nu(t)+\nu^{\dagger}(t)$ and we have assumed perfect detection efficiency. Through the feedback loop, this photocurrent is amplified and used to control the classical field $\varepsilon(t)$. We set

$$
\varepsilon(t)=\frac{g}{2} \int_{0}^{\infty} h(s) I_{\mathrm{hom}}(t-\tau-s) d s,
$$

where $\tau$ is the minimum time delay of the feedback, $h(s)$ is the response function normalized such that $\int_{0}^{\infty} h(s) d s=1$ and $g$ is the round-loop gain (for stability we require $g \operatorname{Re}\left[\tilde{h}(\omega) e^{-i \omega \tau}\right]<1$ for all $\omega$ ) [20].

Solving equation (2.18) in the Fourier domain, where $I_{\text {hom }}(t)$ is given by (2.17), we obtain

$$
\tilde{\varepsilon}(\omega)=\frac{g \tilde{h}(\omega) e^{-i \omega \tau} \tilde{X}_{\nu}(\omega)}{2\left(1-g \tilde{h}(\omega) e^{-i \omega \tau}\right)} .
$$

Thus $b(t)$ has an $X$ quadrature spectrum given by

$$
S_{X}(\omega)=\left|1-g \tilde{h}(\omega) e^{-i \omega \tau}\right|^{-2} .
$$

At frequencies $\bar{\omega}$ well inside the bandwidth of $\tilde{h}(\omega)$ and much less than $\tau^{-1}$, we are in the limit of broadband feedback and can therefore set $\tilde{h}(\bar{\omega}) e^{-i \bar{\omega} \tau}=1$. In this case

$$
S_{X}(\bar{\omega})=(1-g)^{-2} .
$$

Clearly this spectrum is below the standard quantum limit for negative feedback $(g<0)$. For positive feedback, $0<g<1$, we actually increase the noise.

Also, since the feedback acts only on the $X$ quadrature, $S_{Y}(\omega)$ remains at the vacuum limit of unity and

$$
S_{X}(\omega) S_{Y}(\omega)=\left|1-g \tilde{h}(\omega) e^{-i \omega \tau}\right|^{-2},
$$

which can clearly be less than one. This apparent violation of the Heisenberg inequality (2.6) is due to the fact that the in-loop light is not a free field. For time differences $\left|t-t^{\prime}\right|>\tau$, the feedback loop delay time, the usual two-time commutation relations Eq. (2.2) and Eq. (2.4) are are no longer valid, which was first shown by Shapiro et al 14]. Basically this is because parts of the field separated by a time greater than $\tau$ don't actually exist together.

From this we note that non-classical light produced by a feedback loop is not squeezed light in the ordinary sense [3]. First, it is not a free field (as explained above), and second, attempts to remove this light by a beam splitter actually gives above-shot-noise light, as verified experimentally [21,22]. Due to this difference between in-loop light and ordinary squeezed light, it is termed "squashed" light [2,3]. As we will show, while not the 
same as squeezed light, squashed light does have a nonclassical effect on a two-level atom placed within the feedback loop.

Return to Fig. 1 and now include the two-level atom. Again assume that the degree of mode matching of the input light modes to the atom's dipole radiation mode is unity. Then the interaction Hamiltonian has the same form as that given in (2.7), and this linear coupling gives $b^{\prime}(t)=b(t)+\sigma(t)$ [6. Incorporating the feedback as before we find

$$
\tilde{\varepsilon}(\bar{\omega})=\frac{g\left[\tilde{X}_{\nu}(\bar{\omega})+\tilde{\sigma}_{x}(\bar{\omega})\right]}{2(1-g)} .
$$

Again we have assumed the limit of broadband feedback and set $\tilde{h}(\bar{\omega}) e^{-i \bar{\omega} \tau}=1$.

Changing back to the time domain we employ the Markov approximation to state that the feedback is instantaneous in terms of the characteristic response rates of the system. This requires $\bar{\omega}$, still well inside the feedback bandwidth, to be much greater than the atomic linewidth, i.e. $\bar{\omega} \gg 1$. The field incident on the atom is then

$$
b(t)=\nu(t)+\frac{g\left[X_{\nu}\left(t^{-}\right)+\sigma_{x}\left(t^{-}\right)\right]}{2(1-g)},
$$

where the time argument $t^{-}$is used to indicate that even under the Markov approximation the fed-back signal must act after the interaction with the atom.

Substituting $b(t)$ into the interaction Hamiltonian (2.7) gives the Hamiltonian due to the coupling of the atom to the vacuum, plus a feedback Hamiltonian. The vacuum Hamiltonian is just that given in (2.7) except $b(t)$ is replaced by the vacuum operator $\nu(t)$. The feedback Hamiltonian is

$$
H_{\mathrm{fb}}(t)=\frac{\lambda}{2} \sigma_{y}(t)\left[\sigma_{x}\left(t^{-}\right)+X_{\nu}\left(t^{-}\right)\right],
$$

where the feedback parameter $\lambda$ is given by

$$
\lambda=\frac{g}{1-g} \in(-1, \infty)
$$

To describe the evolution generated by this Hamiltonian, we require the Heisenberg-picture theory of homodyne detection and feedback [23]. The result is the following master equation for the atom

$$
\dot{\rho}=\mathcal{D}[\sigma] \rho-i \frac{\lambda}{2}\left[\sigma_{y}, \sigma \rho+\rho \sigma^{\dagger}\right]+\frac{\lambda^{2}}{4} \mathcal{D}\left[\sigma_{y}\right] \rho .
$$

Expressed in terms of $\lambda$, the $X$ quadrature spectrum (2.21) is given by

$$
S_{X}(\bar{\omega})=1+2 \lambda+\lambda^{2}
$$

Thus we can see that if $\lambda<0$ we will squash the $X$ quadrature, i.e. $S_{X}(\bar{\omega})<1$, and the optimal squashing is for $\lambda=-1$.
The master equation again leads to Eqs. (2.11)-(2.13), but this time

$$
\begin{aligned}
& \gamma_{x}=\frac{1}{2}\left(1+2 \lambda+\lambda^{2}\right)=\frac{1}{2} S^{X}(\bar{\omega}), \\
& \gamma_{y}=\frac{1}{2}, \quad \gamma_{z}=\gamma_{x}+\gamma_{y}, \quad C=1+\lambda .
\end{aligned}
$$

Again the decay of $\sigma_{x}$ is inhibited for negative feedback, i.e. for $S_{X}(\bar{\omega})<1$. Most importantly, the dependence of $\gamma_{x}$ on the degree of $X$ quadrature squashing is exactly the same as for squeezing. The only differences between squeezing and squashing are that $\gamma_{y}$ is unaffected by squashing and $C$ is unaffected by squeezing. This follows directly from the squashing of fluctuations which violates the usual uncertainty relations, as discussed following Eq. (2.22) above. Comparing the fluorescence spectra for both cases [5], it can be seen that they are certainly not identical, but both have a sub-natural linewidth.

\section{TWIN-BEAM SQUEEZING}

The master equation for a three-level atom in a broadband squeezed vacuum has previously been developed by Ficek and Drummond [9]. We follow a simplified derivation, based on the same assumptions, which leads to a master equation equivalent to results derived in Part II, Section III of the above reference.

\section{A. Three-level atom master equation}

Ficek and Drummond [9] describe the input field as a broadband squeezed vacuum which is in two-photon resonance with the atom, i.e. $\omega_{0}=\left(\omega_{1}+\omega_{2}\right) / 2$ (where $\omega_{1}$ and $\omega_{2}$ are the atomic transition frequencies). More specifically, they assume the typical squeezing spectrum of a non-degenerate parametric oscillator where the signal and idler fields are tuned to resonance with $\omega_{1}$ and $\omega_{2}$ [8]. Typically, the squeezing bandwidth $B$ is small compared with the carrier frequency $\omega_{0}$.

To simplify the derivation further, we note that if $\omega_{1}$ and $\omega_{2}$ are significantly different, i.e $\left|\omega_{1}-\omega_{2}\right| \gg B$, then the signal and idler fields can be treated as two (distinct) correlated multi-mode fields. Hence, we define the input as a broadband, squeezed twin-beam with the two fields defined by respective continuum annihilation operators $b_{1}(\omega)$ and $b_{2}(\omega)$. Note the squeezed field is broadband in the sense that it appears as $\delta$-correlated squeezed white noise to the atom, i.e. $B \gg \gamma_{1}, \gamma_{2}$, which are the atomic linewidths.

For a broadband squeezed twin-beam with no mean field $\left\langle b_{1}(t)\right\rangle=\left\langle b_{2}(t)\right\rangle=0$, while the correlations are

$$
\begin{aligned}
\left\langle b_{i}^{\dagger}(t) b_{j}\left(t^{\prime}\right)\right\rangle & =N_{i} \delta_{i j} \delta\left(t-t^{\prime}\right), \\
\left\langle b_{i}(t) b_{j}^{\dagger}\left(t^{\prime}\right)\right\rangle & =\left(N_{i}+1\right) \delta_{i j} \delta\left(t-t^{\prime}\right),
\end{aligned}
$$




$$
\begin{aligned}
\left\langle b_{i}(t) b_{j}\left(t^{\prime}\right)\right\rangle & =M\left(1-\delta_{i j}\right) \delta\left(t-t^{\prime}\right), \\
\left\langle b_{i}^{\dagger}(t) b_{j}^{\dagger}\left(t^{\prime}\right)\right\rangle & =M^{*}\left(1-\delta_{i j}\right) \delta\left(t-t^{\prime}\right) .
\end{aligned}
$$

Here $|M|^{2} \leq N_{1} N_{2}+\min \left(N_{1}, N_{2}\right)=N(N+1)$ if $N_{1}=$ $N_{2}=N$, where as before $N$ and $M$ are the broadband approximations of the photon number function (here a doubly peaked function) and the two-photon correlation function. Thus $N_{1}$ and $N_{2}$ have the values of the two peaks in the the photon number function, and correspond to the intensity of each beam. Note again that the $\delta$-functions in these correlations are not exact, their widths being actually of the order $B^{-1}$ (see Sec. II.A).

Consider coupling a three-level atom to the broadband squeezed twin-beam, where the fields $b_{1}$ and $b_{2}$ are coupled to the atomic transitions $|1\rangle \rightarrow|2\rangle$ and $|2\rangle \rightarrow|3\rangle$ respectively. This is shown in Figure 2. Assume again for simplicity that it is possible to mode-match all of the squeezed twin-beam into the atom's input. The Hamiltonian of the atom in the interaction picture at time $t$ is then

$$
\begin{aligned}
H(t)= & i \hbar \sqrt{\gamma_{1}}\left[b_{1}^{\dagger}(t) s_{1}(t)-s_{1}^{\dagger}(t) b_{1}(t)\right] \\
& +i \hbar \sqrt{\gamma_{2}}\left[b_{2}^{\dagger}(t) s_{2}(t)-s_{2}^{\dagger}(t) b_{2}(t)\right],
\end{aligned}
$$

where the atomic parameters are defined in Fig. 2.

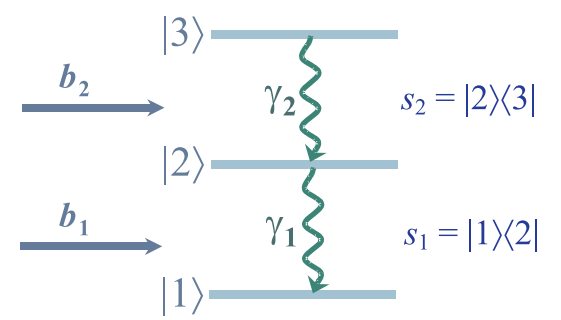

FIG. 2. Coupling of the broadband twin-beam to the three-level atom. The twin-beam is represented by the fields $b_{1}$ and $b_{2}$, while $s_{1}=|1\rangle\langle 2|$ and $s_{2}=|2\rangle\langle 3|$ are the atomic lowering operators and $\gamma_{1}$ and $\gamma_{2}$ are the spontaneous decay rates from levels $|2\rangle$ and $|3\rangle$ respectively.

Applying the unitary evolution of the Hamiltonian (3.2) to an arbitrary system operator $r(t)$ leads to the quantum Langevin equation for the atom (in the Heisenberg picture). Changing from the Heisenberg to the Schrödinger picture is achieved using the relation

$$
\langle d r(t)\rangle=\operatorname{Tr}[d \rho(t) r],
$$

where the picture (Schrödinger or Heisenberg) is specified by the placement of the time argument. The resulting master equation for the atom is

$$
\begin{aligned}
\dot{\rho}= & \left(1+N_{1}\right) \gamma_{1} \mathcal{D}\left[s_{1}\right] \rho+\left(1+N_{2}\right) \gamma_{2} \mathcal{D}\left[s_{2}\right] \rho \\
& +N_{1} \gamma_{1} \mathcal{D}\left[s_{1}^{\dagger}\right] \rho+N_{2} \gamma_{2} \mathcal{D}\left[s_{2}^{\dagger}\right] \rho \\
& +\frac{1}{2} M^{*} \sqrt{\gamma_{1} \gamma_{2}}\left\{\left[s_{1},\left[s_{2}, \rho\right]\right]+\left[s_{2},\left[s_{1}, \rho\right]\right]\right\} \\
& +\frac{1}{2} M \sqrt{\gamma_{1} \gamma_{2}}\left\{\left[s_{1}^{\dagger},\left[s_{2}^{\dagger}, \rho\right]\right]+\left[s_{2}^{\dagger},\left[s_{1}^{\dagger}, \rho\right]\right]\right\}
\end{aligned}
$$

If we set $\gamma_{1}=\gamma_{2}=1, N_{1}=N_{2}=N$ and $M$ real, the master equation reduces to

$$
\begin{gathered}
\dot{\rho}=(1+N)\left\{\mathcal{D}\left[s_{1}\right] \rho+\mathcal{D}\left[s_{2}\right] \rho\right\}+N\left\{\mathcal{D}\left[s_{1}^{\dagger}\right] \rho+\mathcal{D}\left[s_{2}^{\dagger}\right] \rho\right\} \\
+\frac{M}{2}\{[ \\
\left.s_{1},\left[s_{2}, \rho\right]\right]+\left[s_{2},\left[s_{1}, \rho\right]\right]+\left[s_{1}^{\dagger},\left[s_{2}^{\dagger}, \rho\right]\right] \\
\left.+\left[s_{2}^{\dagger},\left[s_{1}^{\dagger}, \rho\right]\right]\right\}
\end{gathered}
$$

The linear versus quadratic dependence of the excited population on intensity, as well as other comparisons of classical and squashed light, are postponed until Sec. V. Again note that for classical noise the evolution of the three-level atom has the same form as (3.5), except $M$ is restricted by $0 \leq|M| \leq N$.

\section{B. Twin-beam quadrature correlations}

From the correlations between the field operators (3.1), we can find the corresponding correlations between the quadratures. The $X$ and $Y$ quadratures are defined as in Eq. (2.3). Then if we define the linear combinations

$$
X^{ \pm}=\frac{1}{\sqrt{2}}\left(X_{2} \pm X_{1}\right), \quad Y^{ \pm}=\frac{1}{\sqrt{2}}\left(Y_{2} \pm Y_{1}\right),
$$

we obtain the following expressions for their non-zero correlations

$$
\begin{aligned}
& \left\langle\tilde{X}^{ \pm}(\omega) X^{ \pm}(0)\right\rangle=1+2 N \pm 2 M, \\
& \left\langle\tilde{Y}^{\mp}(\omega) X^{\mp}(0)\right\rangle=1+2 N \pm 2 M,
\end{aligned}
$$

where we have again set $N_{1}=N_{2}=N$ and $M$ real.

These correlations therefore correspond to the $X$ and $Y$ spectra of the operator combinations $\left\{b_{2}(t) \pm b_{1}(t)\right\} / \sqrt{2}$ [see Eq. (2.5)]. Thus for twin-beam squeezing the squeezed and stretched (depending on the sign of $M$ ) spectra are

$$
\begin{aligned}
& S_{X^{+}}(\omega)=S_{Y^{-}}(\omega)=1+2 N+2 M, \\
& S_{Y^{+}}(\omega)=S_{X^{-}}(\omega)=1+2 N-2 M .
\end{aligned}
$$

That is, assuming $M<0$, the combined field $\left(b_{2}+b_{1}\right) / \sqrt{2}$ has a squeezed $X$ quadrature, while $\left(b_{2}-b_{1}\right) / \sqrt{2}$ has a squeezed $Y$ quadrature.

\section{TWIN-BEAM SQUASHING}

This section develops the critical result of this paper: the master equation of a cascade three-level atom interacting with a squashed twin-beam. This puts us in a position to compare this evolution to that of twin-beam squeezed light and a twin-beam with classical correlations. 


\section{A. Generation of twin-beam squashed light}

Consider the double feedback schematic shown in Fig. 3, but for now ignore the three-level atom. The light beams are shown as double arrows because they represent twin-beams, which are again defined by their continuum annihilation operators, i.e. $\left(b_{1}, b_{2}\right)$. As for twin-beam squeezing the two fields of the squashed twin-beam are noise correlated, where this correlation is now produced by the feedback mechanism (detailed in this section).

Unlike squeezing, here the two fields differ by their polarization rather than their center frequencies (now the same). One beam $b_{1}$ has LHC polarization and the other $b_{2}$ RHC polarization. Thus, linear combinations of the two fields can be easily measured using homodyne detection. In principle the combinations could still be measured if the fields have different frequencies. The drawback in that case is the need for extremely fast detectors whose bandwidth must exceed the frequency difference. For the twin-beam used experimentally [8] this would mean a detector bandwidth of the order of $10^{14} \mathrm{~Hz}$ !

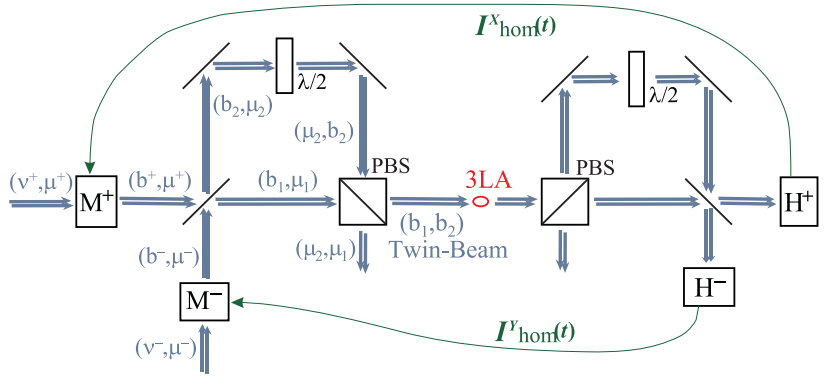

FIG. 3. Experimental configuration for a squashed twin-beam interacting with a three-level atom. The atom is depicted in the center of the figure, coupled to the twin-beam $\left(b_{1}, b_{2}\right)$ as in Fig. 2. The "black box" modulation and detection systems $\mathrm{M}^{+}, \mathrm{M}^{-}, \mathrm{H}^{+}, \mathrm{H}^{-}$, are similar to that detailed in Fig. 1. The $X / Y$ quadrature measured at $\mathrm{H}^{+} / \mathrm{H}^{-}$is used to control the modulation at $\mathrm{M}^{+} / \mathrm{M}^{-}$and in this way produce the squashed correlations between $b_{1}$ and $b_{2}$.

Following Fig. 3, we start on the left side with the input fields $\left(\nu^{+}, \mu^{+}\right)$and $\left(\nu^{-}, \mu^{-}\right)$. These are both twin-beam vacuum fields, where the operators $\nu^{ \pm}(t)$ and $\mu^{ \pm}(t)$ each represent vacuum fluctuations as defined by Eq. (2.16). At this point the two fields of each twin-beam are not correlated; they are two independent vacuum fields. Thus the quadrature operators $X_{\nu}(t)=\nu(t)+\nu^{\dagger}(t)$ and $Y_{\nu}(t)=i \nu^{\dagger}(t)-i \nu(t)$ have statistics described by the spectra $S_{X}(\omega)=S_{Y}(\omega)=1$.

The modulators $\mathrm{M}^{+}, \mathrm{M}^{-}$simply add coherent amplitudes, $\varepsilon^{+}$and $\varepsilon^{-}$, to the LHC components of the input vacuum fields. Both of these "black boxes" are the same as the modulation region depicted in Fig. 1, or see Ref. [5] for details. The LHC fields after the modulators are

$$
b^{+}(t)=\nu^{+}(t)+\varepsilon^{+}(t)
$$

$$
b^{-}(t)=\nu^{-}(t)+i \varepsilon^{-}(t)
$$

where $\varepsilon^{+}(t)$ and $\varepsilon^{-}(t)$ are real functions of time.

The twin-beams now enter a series of optics (a 50:50 beam splitter, perfect mirrors, a $1 / 2$ wave plate and a polarizing beam splitter) to give the desired twin-beam $\left(b_{1}, b_{2}\right)$. The relationships between the continuum annihilation operators $b^{ \pm}$and $b_{i}$ are then

$$
b_{1}=\frac{1}{\sqrt{2}}\left(b^{+}-b^{-}\right), b_{2}=\frac{1}{\sqrt{2}}\left(b^{+}+b^{-}\right) .
$$

It is the purpose of the feedback system to produce nonclassical correlations between $b_{1}$ and $b_{2}$, i.e. to produce the squashed twin-beam $\left(b_{1}, b_{2}\right)$ that will be coupled to the three-level atom.

In the previous section, III.B, on squeezed twin-beams, equation (3.9) shows that the $X$ quadrature of $\left(b_{1}+\right.$ $\left.b_{2}\right) / \sqrt{2}$ and the $Y$ quadrature of $\left(b_{2}-b_{1}\right) / \sqrt{2}$ are squeezed for $M<0$, i.e. $S_{X^{+}}=S_{Y^{-}}<1$. For the generation of our squashed twin-beam we require the same quadratures to have squashed correlations. From the above relationship (4.3), it can be seen that the $X$ quadrature of $b^{+}$ and the $Y$ quadrature of $b^{-}$give these combinations.

Therefore, we reverse the optical setup on the other side of the atom. In the absence of the atom, the same fields $b^{+}(t), b^{-}(t)$ will enter the homodyne detection devices $\mathrm{H}^{+}, \mathrm{H}^{-}$, which are set up to measure the $X$ quadrature of $b^{+}$and the $Y$ quadrature of $b^{-}$. These "black box" devices are again the same as that depicted in Fig. 1. Note that $\mathrm{M}^{+}$and $\mathrm{H}^{+}$(and $\mathrm{M}^{-}$and $\mathrm{H}^{-}$) are really coupled by a shared local oscillator, which in this case is a large LHC polarized coherent field.

Again assuming perfect detection, we obtain for the homodyne photocurrents

$$
\begin{aligned}
& I_{\mathrm{hom}}^{X}(t)=X_{\nu}^{+}(t)+2 \varepsilon^{+}(t), \\
& I_{\mathrm{hom}}^{Y}(t)=Y_{\nu}^{-}(t)+2 \varepsilon^{-}(t) .
\end{aligned}
$$

Through the two feedback loops, these currents may be used to determine the classical field amplitudes $\varepsilon^{+}(t)$ and $\varepsilon^{-}(t)$ :

$$
\begin{aligned}
& \varepsilon^{+}(t)=\frac{g}{2} \int_{0}^{\infty} h(s) I_{h o m}^{X}(t-\tau-s) d s, \\
& \varepsilon^{-}(t)=\frac{g}{2} \int_{0}^{\infty} h(s) I_{h o m}^{Y}(t-\tau-s) d s
\end{aligned}
$$

where we have assumed the same characteristics for both feedback loops.

Solving in the Fourier domain and substituting into equations (4.1) and (4.2) yields

$$
\begin{aligned}
& \tilde{b}^{+}(\omega)=\tilde{\nu}^{+}(\omega)+\frac{g \tilde{h}(\omega) e^{-i \omega \tau} \tilde{X}_{\nu^{+}}(\omega)}{2\left[1-g \tilde{h}(\omega) e^{-i \omega \tau}\right]}, \\
& \tilde{b}^{-}(\omega)=\tilde{\nu}^{-}(\omega)+i \frac{g \tilde{h}(\omega) e^{-i \omega \tau} \tilde{Y}_{\nu^{-}}(\omega)}{2\left[1-g \tilde{h}(\omega) e^{-i \omega \tau}\right]} .
\end{aligned}
$$


The $X$ quadrature spectrum of $b^{+}$and the $Y$ quadrature spectrum of $b^{-}$are then

$$
\begin{aligned}
& S_{X^{+}}(\omega)=\left|1-g \tilde{h}(\omega) e^{-i \omega \tau}\right|^{-2}, \\
& S_{Y^{-}}(\omega)=\left|1-g \tilde{h}(\omega) e^{-i \omega \tau}\right|^{-2} .
\end{aligned}
$$

Assuming again the limit of broadband feedback, we set $\tilde{h}(\bar{\omega}) e^{-i \bar{\omega} \tau}=1$ to give

$$
S_{X^{+}}(\bar{\omega})=S_{Y^{-}}(\bar{\omega})=(1-g)^{-2} .
$$

As before these spectra are below the standard quantum limit for negative feedback, i.e. for $g<0$.

Therefore, the $X$ quadrature of $\left(b_{1}+b_{2}\right) / \sqrt{2}$ and the $Y$ quadrature of $\left(b_{2}-b_{1}\right) / \sqrt{2}$ are squashed as required, and we can now model the effects produced by a squashed twin-beam $\left(b_{1}, b_{2}\right)$ interacting with a three-level atom.

\section{B. Inclusion of the three-level atom}

Return to Fig. 3 and now include the three-level atom. Assume for simplicity that it is possible to mode-match all of $\left(b_{1}, b_{2}\right)$ into the atom's input. Also, assume that the three energy levels, $|1\rangle,|2\rangle$ and $|3\rangle$, are equally spaced and have magnetic quantum numbers, $m_{j}$, of 0,1 , and 0 respectively. This means that $b_{1}$ (with LHC polarization) will couple to the $|1\rangle \rightarrow|2\rangle$ transition and $b_{2}$ (with RHC polarization) will couple to the $|2\rangle \rightarrow|3\rangle$ transition, as shown in Fig. 2.

The interaction Hamiltonian is the same as that given for twin-beam squeezing (3.2). From here on we will set $\gamma_{1}=\gamma_{2}=1$. Under a linear coupling as in (3.2), $b_{1}^{\prime}(t)=b_{1}(t)+s_{1}(t)$ and $b_{2}^{\prime}(t)=b_{2}(t)+s_{2}(t)$. Thus the the homodyne photocurrents (4.4) and (4.5) become

$$
\begin{gathered}
I_{\mathrm{hom}}^{X}(t)+\frac{1}{\sqrt{2}}\left[s_{1}^{x}(t)+s_{2}^{x}(t)\right], \\
I_{\mathrm{hom}}^{Y}(t)+\frac{1}{\sqrt{2}}\left[s_{1}^{y}(t)-s_{2}^{y}(t)\right],
\end{gathered}
$$

where $s^{x}=s+s^{\dagger}$ and $s^{y}=i s-i s^{\dagger}$.

Incorporating the feedback as before we solve (4.6) and (4.7) in the Fourier domain. We obtain similar expressions for $\tilde{b}^{+}(\omega)$ and $\tilde{b}^{-}(\omega)$ as those given in Eqs. (4.8, 4.9). The atom-field coupling simply adds the term $\left[\tilde{s}_{1}^{x}(\omega)+\tilde{s}_{2}^{x}(\omega)\right] / \sqrt{2}$ to $\tilde{X}_{\nu^{+}}(\omega)$ and the term $\left[\tilde{s}_{1}^{y}(\omega)-\tilde{s}_{2}^{y}(\omega)\right] / \sqrt{2}$ to $\tilde{Y}_{\nu^{-}}(\omega)$. Note that the terms $\tilde{\nu}^{+}(\omega)$ and $\tilde{\nu}^{-}(\omega)$ are unaffected by this substitution.

In the limit of broadband feedback (refer to Section II.B. for the details of the Markov approximation), the field operators acting on the atom are then

$$
\begin{aligned}
b_{1}(t)= & {\left[\nu^{+}(t)-\nu^{-}(t)\right] / \sqrt{2} } \\
& +\frac{g\left\{X_{\nu^{+}}\left(t^{-}\right)+\left[s_{1}^{x}\left(t^{-}\right)+s_{2}^{x}\left(t^{-}\right)\right] / \sqrt{2}\right\}}{2(1-g) \sqrt{2}}
\end{aligned}
$$

$$
\begin{aligned}
& -i \frac{g\left\{Y_{\nu^{-}}\left(t^{-}\right)+\left[s_{1}^{y}\left(t^{-}\right)-s_{2}^{y}\left(t^{-}\right)\right] / \sqrt{2}\right\}}{2(1-g) \sqrt{2}}, \\
b_{2}(t)= & {\left[\nu^{+}(t)+\nu^{-}(t)\right] / \sqrt{2} } \\
& +\frac{g\left\{X_{\nu^{+}}\left(t^{-}\right)+\left[s_{1}^{x}\left(t^{-}\right)+s_{2}^{x}\left(t^{-}\right)\right] / \sqrt{2}\right\}}{2(1-g) \sqrt{2}} \\
& +i \frac{g\left\{Y_{\nu^{-}}\left(t^{-}\right)+\left[s_{1}^{y}\left(t^{-}\right)-s_{2}^{y}\left(t^{-}\right)\right] / \sqrt{2}\right\}}{2(1-g) \sqrt{2}},
\end{aligned}
$$

where the relation (4.3) has been used. Here the time argument $t^{-}$is again used to indicate that the fed-back signal is infinitesimally delayed from the vacuum noise.

The total interaction Hamiltonian can be written

$$
H(t)=H_{0}(t)+H_{\mathrm{fb}}(t),
$$

where $H_{0}(t)$ is the same as in (3.2) assuming no feedback, that is

$$
\begin{aligned}
H_{0}(t)= & \frac{i}{\sqrt{2}}\left[\nu^{+}(t)-\nu^{-}(t)\right]^{\dagger} s_{1}(t)+\text { H.c } \\
& +\frac{i}{\sqrt{2}}\left[\nu^{+}(t)+\nu^{-}(t)\right]^{\dagger} s_{2}(t)+\text { H.c. }
\end{aligned}
$$

Substituting $b_{1}(t)$ and $b_{2}(t)$ into (3.2), we find for the feedback Hamiltonian

$$
H_{\mathrm{fb}}(t)=F_{X}(t) X_{\nu^{+}}^{\prime}\left(t^{-}\right)+F_{Y}(t) Y_{\nu^{-}}^{\prime}\left(t^{-}\right),
$$

where the quadrature operators are given by

$$
\begin{aligned}
X_{\nu^{+}}^{\prime}\left(t^{-}\right) & =X_{\nu^{+}}\left(t^{-}\right)+\left[s_{1}^{x}\left(t^{-}\right)+s_{2}^{x}\left(t^{-}\right)\right] / \sqrt{2}, \\
Y_{\nu^{-}}^{\prime}\left(t^{-}\right) & =Y_{\nu^{-}}\left(t^{-}\right)+\left[s_{1}^{y}\left(t^{-}\right)-s_{2}^{y}\left(t^{-}\right)\right] / \sqrt{2},
\end{aligned}
$$

and the feedback operators are

$$
\begin{aligned}
& F_{X}(t)=\frac{\lambda}{2}\left[s_{1}^{y}(t)+s_{2}^{y}(t)\right] / \sqrt{2}, \\
& F_{Y}(t)=\frac{\lambda}{2}\left[s_{2}^{x}(t)-s_{1}^{x}(t)\right] / \sqrt{2} .
\end{aligned}
$$

Here we have again used the feedback parameter $\lambda$, defined in Eq. (2.26). The infinitesimal time delay denoted by $t^{-}$ensures that the quadrature operators $X_{\nu^{+}}^{\prime}\left(t^{-}\right)$and $Y_{\nu^{-}}^{\prime}\left(t^{-}\right)$commute with all system operators at the same time $t$ 23. Therefore they will commute with $F_{X}(t)$ and $F_{Y}(t)$ and there is no ordering ambiguity in (4.19).

The evolution of an arbitrary system operator $r(t)$ generated by (4.19) is

$$
\begin{aligned}
{[d r]_{\mathrm{fb}}=} & U_{\mathrm{fb}}^{\dagger} r U_{\mathrm{fb}}-r \\
= & i X_{\nu^{+}}^{\prime}\left(t^{-}\right) d t\left[F_{X}, r\right]-\frac{1}{2}\left[F_{X},\left[F_{X}, r\right]\right] d t \\
& +i Y_{\nu^{-}}^{\prime}\left(t^{-}\right) d t\left[F_{Y}, r\right]-\frac{1}{2}\left[F_{Y},\left[F_{Y}, r\right]\right] d t .
\end{aligned}
$$

where all time arguments are $t$ unless otherwise given, and the unitary evolution operator is defined as $U_{\mathrm{fb}}=$ $\exp \left[-i H_{\mathrm{fb}} d t\right]$. 
Adding in the non-feedback evolution gives the total Langevin equation $d r$, which following the process of 23] leads to total master equation for the atom. This involves separating $X_{\nu^{+}}^{\prime}\left(t^{-}\right) d t$ such that $\nu^{\prime+}\left(t^{-}\right) d t$ (which equals $\left.\nu^{+}\left(t^{-}\right) d t+\left[s_{1}\left(t^{-}\right)+s_{2}\left(t^{-}\right)\right] d t / \sqrt{2}\right)$ is moved to the right side and $\left[\nu^{\prime+}\left(t^{-}\right)\right]^{\dagger} d t$ is moved to the left side of $\left[F_{X}, r\right]$ (using the fact that $\nu^{\prime \pm}\left(t^{-}\right)$commute with system operators at the same time). The same is done for $Y_{\nu^{+}}^{\prime}\left(t^{-}\right)$.

The operators $\nu^{\prime}\left(t^{-}\right)$are now in the correct position to annihilate the vacuum when the trace over the bath density operator is taken. Taking the total trace over the system and bath density operators gives $\langle d r\rangle$, and here we can ignore the time difference since for arbitrary system operators $a(t)$ and $b(t)$

$$
\lim _{\tau \rightarrow 0}\langle a(t) b(t-\tau)\rangle=\langle a(t) b(t)\rangle .
$$

The final step involves changing from the Heisenberg to the Schrödinger picture and deriving the evolution of the density operator for the atom alone. As noted previously, this is found from Eq. (3.3).

The resultant master equation for a cascade three-level atom interacting with twin-beam squashed light is

$$
\begin{aligned}
\dot{\rho}= & \left(1+\lambda+\frac{\lambda^{2}}{4}\right)\left\{\mathcal{D}\left[s_{1}\right] \rho+\mathcal{D}\left[s_{2}\right] \rho\right\} \\
& +\frac{\lambda^{2}}{4}\left\{\mathcal{D}\left[s_{1}^{\dagger}\right] \rho+\mathcal{D}\left[s_{2}^{\dagger}\right] \rho\right\} \\
& +\frac{\lambda}{2}\left\{\left[s_{1},\left[s_{2}, \rho\right]\right]+\left[s_{1}^{\dagger},\left[s_{2}^{\dagger}, \rho\right]\right]\right\} \\
& +\frac{\lambda^{2}}{8}\left\{\left[s_{1},\left[s_{2}, \rho\right]\right]+\left[s_{1}^{\dagger},\left[s_{2}^{\dagger}, \rho\right]\right]\right. \\
& \left.\quad+\left[s_{2},\left[s_{1}, \rho\right]\right]+\left[s_{2}^{\dagger},\left[s_{1}^{\dagger}, \rho\right]\right]\right\} .
\end{aligned}
$$

The spectra for the squashed quadratures were given in Eq. (4.12). We can see that in terms of the feedback parameter $\lambda$ they have the same form as the squashed spectra for the single squashed beam in Section II.C. This is given by Eq. (2.28). Note that the spectra are only squashed for $-1<\lambda<0$. These quadrature correlations can be re-expressed in terms of the field operators $b_{1}$ and $b_{2}$. We find that the non-zero correlations for squashing are $(i \neq j)$

$$
\begin{aligned}
\left\langle b_{i}^{\dagger}(t) b_{i}\left(t^{\prime}\right)\right\rangle & =\frac{\lambda^{2}}{4} \delta\left(t-t^{\prime}\right), \\
\left\langle b_{i}(t) b_{i}^{\dagger}\left(t^{\prime}\right)\right\rangle & =\left(1+\lambda+\frac{\lambda^{2}}{4}\right) \delta\left(t-t^{\prime}\right), \\
\left\langle b_{i}(t) b_{j}\left(t^{\prime}\right)\right\rangle & =\left(\frac{\lambda}{2}+\frac{\lambda^{2}}{4}\right) \delta\left(t-t^{\prime}\right), \\
\left\langle b_{i}^{\dagger}(t) b_{j}^{\dagger}\left(t^{\prime}\right)\right\rangle & =\left(\frac{\lambda}{2}+\frac{\lambda^{2}}{4}\right) \delta\left(t-t^{\prime}\right) .
\end{aligned}
$$

Again the $\delta$-functions here are not exact. Their width is of the order $B^{-1}$ where $B \gg 1$ is the squashing band- width. Note the modified commutation relations implied by the first two equations here, i.e. $\left[b_{i}(t), b_{i}^{\dagger}\left(t^{\prime}\right)\right] \neq$ $\delta\left(t-t^{\prime}\right)$.

If we had constructed our feedback mechanism such that we squashed the $Y^{+}$and $X^{-}$quadratures rather than $X^{+}$and $Y^{-}$, then the correlations would be same as in (4.25) except $\left\langle b_{i}(t) b_{j}\left(t^{\prime}\right)\right\rangle$ and $\left\langle b_{i}^{\dagger}(t) b_{j}^{\dagger}\left(t^{\prime}\right)\right\rangle$ would be negative. Correspondingly, the master equation for the atom would be the same as Eq. (4.24) except the last two terms $\left(\lambda / 2\right.$ and $\left.\lambda^{2} / 8\right)$ would be negative.

\section{COMPARISON OF SQUASHED LIGHT WITH SQUEEZED AND CLASSICAL LIGHT}

This section collects the main results of the previous sections, which are the correlations of squeezed, squashed and classical light, and the resulting master equations due to the interaction of this light with two- and threelevel atoms. We find that that a unified formalism can be found in terms of effective $N$ and $M$ parameters. The master equation for squashed light is then compared to those for squeezed and classical light in order to determine the non-classical nature of squashed light.

\section{A. Unified formalism}

The general formalism for broadband squeezed, squashed and classical light interacting with simple atomic systems involves effective $N$ and $M$ parameters. For squeezed and classical fields, these values are the usual broadband limits of the photon number function and the two-photon correlation function. For squashed fields it is more complicated. Due to the fact that it is not a free field (see Section II.B.), the correlations of squashed light need to be separated into upward and downward processes.

The most general parameters for the three types of light are $N_{U}, N_{D}, M_{U}$ and $M_{D}$, where the $U$ and $D$ subscripts refer to upward (excitation) and downward (deexcitation) processes. The two-level atom master equations for squeezing (2.9) and squashing 2.27 can both be rewritten in terms of these parameters to give the same general master equation. It is

$$
\begin{aligned}
\dot{\rho}= & \left(1+N_{D}\right) \mathcal{D}[\sigma] \rho+N_{U} \mathcal{D}\left[\sigma^{\dagger}\right] \rho \\
& -\frac{1}{2}\left(M_{D}+M_{U}\right)\left\{\sigma \rho \sigma+\sigma^{\dagger} \rho \sigma^{\dagger}\right\} .
\end{aligned}
$$

For the three-level atom, the general master equation is obtained by unifying Eqs. (3.5) and (4.24). The result is

$$
\begin{aligned}
\dot{\rho}= & \left(1+N_{D}\right)\left\{\mathcal{D}\left[s_{1}\right] \rho+\mathcal{D}\left[s_{2}\right] \rho\right\}+N_{U}\left\{\mathcal{D}\left[s_{1}^{\dagger}\right] \rho+\mathcal{D}\left[s_{2}^{\dagger}\right] \rho\right\} \\
& +\frac{1}{2} M_{D}\left\{\left[s_{1},\left[s_{2}, \rho\right]\right]+\left[s_{1}^{\dagger},\left[s_{2}^{\dagger}, \rho\right]\right]\right\} \\
& +\frac{1}{2} M_{U}\left\{\left[s_{2},\left[s_{1}, \rho\right]\right]+\left[s_{2}^{\dagger},\left[s_{1}^{\dagger}, \rho\right]\right]\right\} .
\end{aligned}
$$


The effective $N$ and $M$ parameters are the same for both master equations. For squashed light they are

$$
\begin{array}{ll}
N_{U}=\lambda^{2} / 4, & N_{D}=\lambda+\lambda^{2} / 4, \\
M_{U}= \pm N_{U}, & M_{D}= \pm N_{D},
\end{array}
$$

where $-1<\lambda<0$. Here the + refers to measuring the $X^{+}$and $Y^{-}$quadratures and vice versa for the - , as explained at the end of the preceding section. For maximally squeezed light we have

$$
\begin{aligned}
& N_{U}=N_{D}=N, \\
& M_{U}=M_{D}=\mp \sqrt{N(N+1)},
\end{aligned}
$$

and for maximally correlated classical light

$$
\begin{aligned}
& N_{U}=N_{D}=N, \\
& M_{U}=M_{D}=\mp N .
\end{aligned}
$$

In all three cases, the sign of $M$ indicates which quadratures are squashed (below-shot-noise spectra), squeezed (below-shot-noise spectra) or classically noisy (above-shot-noise spectra). For the single field the $X$ and $Y$ quadrature spectra are

$$
\begin{aligned}
& S_{X}(\omega)=1+N_{U}+N_{D}+M_{U}+M_{D}, \\
& S_{Y}(\omega)=1+N_{U}+N_{D}-M_{U}-M_{D},
\end{aligned}
$$

while for the twin-beam we have

$$
\begin{aligned}
& S_{X^{+}}(\omega)=S_{Y^{-}}(\omega)=1+N_{U}+N_{D}+M_{U}+M_{D}, \\
& S_{X^{-}}(\omega)=S_{Y^{+}}(\omega)=1+N_{U}+N_{D}-M_{U}-M_{D} .
\end{aligned}
$$

Thus, to clarify the above equations, if $M_{U / D}=$ $+N_{U / D}$ for squashed light, then $S_{X}=S_{X^{+}}=S_{Y^{-}}<1$ (since $\lambda<0$ ) while the conjugate quadratures $S_{Y}=$ $S_{Y^{+}}=S_{X^{-}}=1$. If $M_{U / D}=-N_{U / D}$ the reverse is true. These results are the same for classical light except now the affected spectra are above unity, i.e. if $M=N$ then $S_{X}=S_{X^{+}}=S_{Y^{-}}>1$ while $S_{Y}=S_{Y^{+}}=$ $S_{X^{-}}=1$, and vice versa for $M=-N$. For squeezed light we have the expected Heisenberg trade-off. That is, if $M=\sqrt{N(N+1)}$ then $S_{X}=S_{X^{+}}=S_{Y^{-}}>1$ while $S_{Y}=S_{Y^{+}}=S_{X^{-}}<1$, and vice versa for $M=-\sqrt{N(N+1)}$.

\section{B. Effective $N$ and $M$ parameters}

The values for $N_{U}, N_{D}$ and $M_{\mathrm{av}}=\left(M_{U}+M_{D}\right) / 2$ can be simply related to the field correlations. For a single broadband squashed (or squeezed or classical) field these are

$$
\begin{aligned}
\left\langle b^{\dagger}(t) b\left(t^{\prime}\right)\right\rangle & =N_{U} \delta\left(t-t^{\prime}\right), \\
\left\langle b(t) b^{\dagger}\left(t^{\prime}\right)\right\rangle & =\left(1+N_{D}\right) \delta\left(t-t^{\prime}\right), \\
\left\langle b(t) b\left(t^{\prime}\right)\right\rangle & =\left\langle b^{\dagger}(t) b^{\dagger}\left(t^{\prime}\right)\right\rangle=M_{\mathrm{av}} \delta\left(t-t^{\prime}\right),
\end{aligned}
$$

The unified correlations for the twin-beam combine Eqs. (3.1) and (4.25) and are given by $(i \neq j)$

$$
\begin{aligned}
\left\langle b_{i}(t)^{\dagger} b_{i}\left(t^{\prime}\right)\right\rangle & =N_{U} \delta\left(t-t^{\prime}\right), \\
\left\langle b_{i}(t) b_{i}^{\dagger}\left(t^{\prime}\right)\right\rangle & =\left(1+N_{D}\right) \delta\left(t-t^{\prime}\right), \\
\left\langle b_{i}(t) b_{j}\left(t^{\prime}\right)\right\rangle & =\left\langle b_{i}^{\dagger}(t) b_{j}^{\dagger}\left(t^{\prime}\right)\right\rangle=M_{\mathrm{av}} \delta\left(t-t^{\prime}\right) .
\end{aligned}
$$

The individual choices for $M_{U}$ and $M_{D}$ are made apparent when the master equations for the three-level atom is broken into upwards and downwards transitions. This is best illustrated by expressing the general master equation in diagrammatic form. Fig. 4 shows the diagrammatic master equation for the three-level atom, where $\rho_{13}$ (which equals $\rho_{31}$ for real $M$ ) is the two-photon coherence between the upper and lower states.

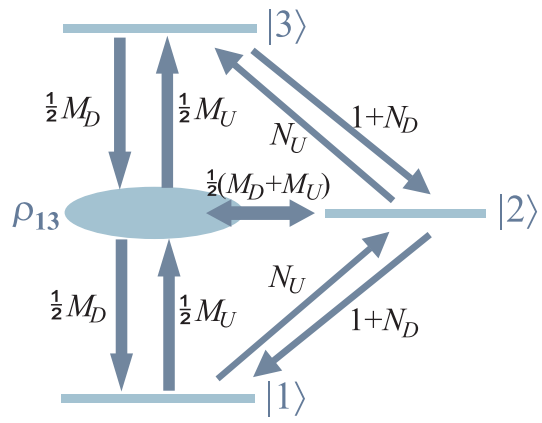

FIG. 4. Diagrammatic master equation for a cascade three-level atom interacting with a broadband field. The levels are labelled by $|1\rangle,|2\rangle$ and $|3\rangle$, while the two-photon coherence is given by $\rho_{13}$. The transition rates, indicated by the arrows, are given by the effective $N$ and $M$ parameters.

The transition rates in this diagram are found by reexpressing the master equation in terms of the state vectors $|1\rangle,|2\rangle$ and $|3\rangle$ and using the matrix representation for the density operator. That is

$$
\rho=\sum_{i, j=1}^{3} \rho_{i j}|i\rangle\langle j|,
$$

where the matrix elements for $i=j$ are the occupation probabilities and those for $i \neq j$ are the coherences. We find two closed sets of coupled differential equations for $\rho_{i j}$. The first shows that $\rho_{12}=\rho_{21}=\rho_{23}=\rho_{32}=0$. The second set contains the equations of motion for the three populations $\rho_{i i}$ and the two-photon coherence $\rho_{13}$. These coupled equations give the transition rates seen in Fig. 4.

By simply comparing the correlation parameters, squashed light appears classical in the sense that $|M|=$ $N$. Yet the squashed spectra, e.g. $S_{X}=1+2 \lambda+\lambda^{2}$, are below the standard quantum limit of unity for $-1<\lambda<$ 0 which is clearly non-classical. For the spectra, terms involving $\lambda$ can thus be thought of as the non-classical terms, while terms involving $\lambda^{2}$ are classical. 
Looking at the master equation (see Fig. 4) we see that this non-classical term also appears in the de-excitation process since $N_{D}=M_{D}=\lambda+\lambda^{2} / 4$, whereas the excitation process appears classical in all regards since here $N_{U}=M_{U}=\lambda^{2} / 4$. Therefore, we can anticipate that the non-classical two-photon excitation seen for squeezed light might not be seen for squashed light. However, we can also expect there to be a corresponding non-classical process in the de-excitation.

\section{Basis for comparison}

The features of the master equations for squashed, squeezed and classical light can now be compared. Here we address the question of what to keep constant between these three cases. In the past, squeezed and classical light have been compared in terms of the intensity since it is defined in the same way for both types of light. The most obvious definition for the intensity $\mathcal{N}$ is in terms of the photon flux operator, i.e.

$$
\left\langle b_{i}^{\dagger}(t) b_{i}\left(t^{\prime}\right)\right\rangle=\mathcal{N} \delta\left(t-t^{\prime}\right)
$$

If we extend this definition of intensity to include squashed light, we must not forget that, in this case, commuting the operators does not change $\mathcal{N}$ into $1+\mathcal{N}$. Therefore the term "intensity" does not have the same meaning for squashed light. Nevertheless, the parameter $\mathcal{N}$ is the most natural choice as we will see below.

Applying this definition of intensity (5.13) for all three types of light we see that, in general, $\mathcal{N}=N_{U}$. For the squeezed and classical fields this simply means that $N=\mathcal{N}$ and $|M|=\sqrt{\mathcal{N}(\mathcal{N}+1)}$ for squeezing, and $|M|=\mathcal{N}$ for classical light. For squashed light the relationship is $\lambda=-2 \sqrt{\mathcal{N}}$. Since the spectra (5.6) and 5.8 ) are only below unity for $-1<\lambda<0$ (negative feedback), the "intensity" range for squashed light is thus limited to $0<\mathcal{N}<0.25$.

It is interesting to note that the feedback described in Sections II.B and IV can be modified to produce classical light which has the same intensity as the squashed light. This would enable experimental comparison of squashed and classical light to be made with the same apparatus. As opposed to using positive feedback $g>0$, which increases the noise in the fed-back quadratures, the method introduced here (see Fig. 5) actually produces maximally correlated classical light, i.e. $N_{U}=N_{D}=N$ and $M_{U}=M_{D}=N$.

Consider the simplified diagram for producing squashed light seen in Fig. 5(a). This theory is detailed in [5, 3] and is also outlined in Section II.B. Now, by simply separating the feedback loop from the test system, as shown in Fig. 5(b), it can be shown that the light, $a(t)$, interacting with the system is then maximally correlated classical noise. Note that it is a relatively simple matter to extend this for the twin-beam case.
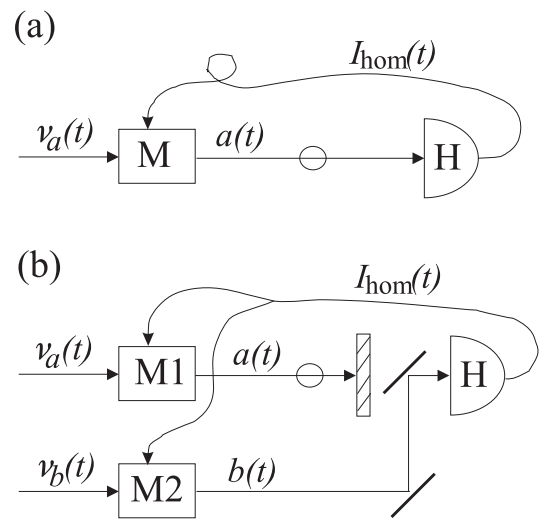

FIG. 5. Simplified diagrams for (a) the production of squashed light and (b) the production of maximally correlated classical light using feedback. The circles are where a test atom could be positioned.

Here we start with two initial vacuum fields $\nu_{a}$ and $\nu_{b}$ to which coherent amplitudes are again added (by M1 and M2) resulting in the fields $a$ and $b$. However, only the $X$ quadrature of $b$ is measured and used to control the same coherent amplitude, $\varepsilon$, added to both the initial vacuum fields. That is, $a=\nu_{a}+\varepsilon$ and $b=\nu_{b}+\varepsilon$. Assuming the same modulation as in Eq. (2.18), we obtain in the broadband approximation

$$
a(t)=\nu_{a}(t)+\frac{g X_{b}\left(t^{-}\right)}{2(1-g)} .
$$

Coupling a two-level atom this field with perfect modematching again leads to the general master equation (5.1) except now

$$
\begin{aligned}
& N_{U}=\mathcal{N}=N_{D}=\lambda^{2} / 4 \\
& M_{U}=M_{D}=\lambda^{2} / 4 .
\end{aligned}
$$

Therefore, by comparison with Eqs. (5.5), we see that this is maximally correlated classical light with the same intensity as squashed light (5.3).

Note that here $M$ is positive which means that $S_{X}>1$ and $S_{Y}=1$, which can also be noted from the fact that we are adding noise to the $X$ quadrature of $a$ (5.14). To set $S_{X}=1$ and $S_{Y}>1$ we would need to measure the $Y$ quadrature of $b$ and use it to add noise to the $Y$ quadrature of $a$.

By choosing a fixed value for $\mathcal{N}$ we are able to make a phase space comparison between squashed, squeezed and classical light. This is shown in Fig. 6 for $\mathcal{N}=0.1$, where we have chosen $M_{U / D}=N_{U / D}$ for squashed light and $M<0$ for squeezed and classical light. We emphasize that these are not the contours of a Wigner function of a single-mode harmonic oscillator. All the fields discussed in this paper are multi-mode fields denoted by continuum annihilation operators (see Secs. II.A and III.A). In particular, squashing has no meaning for a single-mode field. Figure 6 is best interpreted as a radial plot, where the 
distance of the curve from the origin at an angle $\theta$ represent the broadband values of the quadrature spectra, $S_{Q_{\theta}}(\bar{\omega})$.

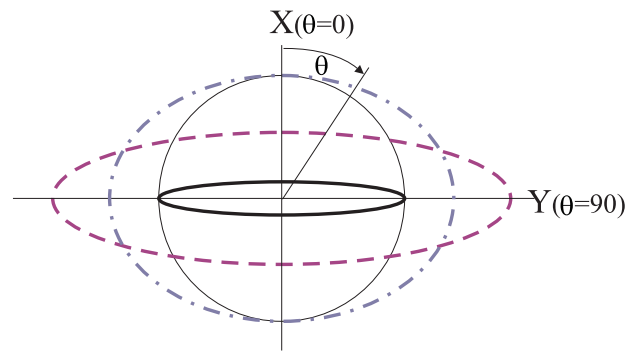

FIG. 6. Phase space comparison between squashed, squeezed and classical light, where the intensity $\mathcal{N}=0.1$ is kept fixed for all three cases. The contours represent the value of $S_{Q_{\theta}}(\bar{\omega})$, where $Q_{\theta}=X \cos \theta+Y \sin \theta$. The circle represents the vacuum state, the solid ellipse is the squashed state, the dashed ellipse is the squeezed state and the dash-dot ellipse represents the classical noise state.

This figure highlights the difference between squashed and squeezed light that was discussed previously in Sec. II.B. While both are non-classical fields in the sense of reduced fluctuations in the $X$ quadrature, squashed light does not have the corresponding increase in the $Y$ quadrature. Hence, the fluctuations are simply squashed from the vacuum limit in one quadrature, as opposed to squeezed from one quadrature into the other.

For the rest of the paper we will assume correlations like that given in Fig. 6. That is, $M_{U / D}=N_{U / D}\left(S_{X}=\right.$ $\left.S_{X^{+}}=S_{Y^{-}}<1\right)$ for squashed light, $M=-\sqrt{N(N+1)}$ $\left(S_{X}=S_{X^{+}}=S_{Y^{-}}<1\right)$ for squeezed light, and $M=-N$ $\left(S_{X}=S_{X^{+}}=S_{Y^{-}}=1\right)$ for classical light.

\section{Steady state excited populations}

The squeezed master equation leads to a steady state excited population with a linear (non-classical), as well as quadratic (classical), dependence on the intensity. This has been shown both theoretically [9,10] and experimentally [8].

Normal (classical) two-photon excitation involves a two-step process with one photon absorbed in each step (hence the quadratic dependence). Squeezed light has large two-photon correlations and therefore the excitation from the ground to the upper state can occur in one step. We have shown that squashed light also has non-classical two-photon correlations as evidenced by the below-shot-noise spectrum (2.28). However, squashed light does not produce an excited population with a linear dependence on the intensity, as shown below.

The steady state population of the upper state for the twin-beam squeezed vacuum field is

$$
\rho_{33}=\frac{\mathcal{N}}{1+2 \mathcal{N}} \simeq \mathcal{N} .
$$

The corresponding result for a twin-beam with classical noise is

$$
\rho_{33}=\frac{2 \mathcal{N}^{2}}{(1+2 \mathcal{N})(1+3 \mathcal{N})} \simeq 2 \mathcal{N}^{2} .
$$

Here the asymptotic values are for the low intensity regime, $\mathcal{N} \ll 1$. The first equation shows the linear dependence on $\mathcal{N}$ that is characteristic of squeezed light, whereas the corresponding excited population for classical light has a quadratic dependence on $\mathcal{N}$. The exact equation for squeezed light also has a quadratic dependence on $\mathcal{N}$ for imperfect mode-matching (not included in our analysis).

The excited population for twin-beam squashed light is given by

$$
\rho_{33}=\frac{2 \mathcal{N}^{2}}{1-\sqrt{\mathcal{N}}(6+10 \mathcal{N})+\mathcal{N}(13+6 \mathcal{N})} \simeq 2 \mathcal{N}^{2},
$$

For low intensities, $\sqrt{\mathcal{N}} \ll 1$ (which really corresponds to weak feedback $|\lambda| \ll 1$ ), this equation is clearly classical with a quadratic dependence on $\mathcal{N}$. Thus, we see that squashed light fails to give a non-classical excited population similar to squeezed light.

\section{E. Transient two-photon coherences}

We have shown that squashed light excitation of a three-level atom is a classical process. This can be understood by looking at the two-photon transition rate for excitation, $M_{U}=\lambda^{2} / 4$. As we pointed out earlier $\lambda^{2}$ corresponds to the classical term in the squashed spectrum (equal to $1+2 \lambda+\lambda^{2}$ ). It is the two-photon $d e-$ excitation rate that has the non-classical dependence on $\lambda$, i.e. $M_{D}=\lambda+\lambda^{2} / 4$.

This section will show that squashed light de-excitation of a three-level atom is non-classical. However, it only behaves similarly to squeezed light early during the deexcitation and then only for weak feedback. This can be seen in the transient coherence $\rho_{13}$ between the upper and lower levels. In the short time regime, $t \ll 1$, the coherence will have only received population from the initial state, $|3\rangle$. For all three types of light (squashed, squeezed and classical), the transient coherence from the upper level is

$$
\rho_{13} \simeq \frac{M_{D}}{2} t
$$

which can be seen in Fig. 4.

To compare the coherences in terms of the intensity of the input light (squashed, squeezed or classical) we simply substitute the expressions for $M_{D}$ in terms of the respective intensities. For squashed light this means setting $M_{D}=-2 \sqrt{\mathcal{N}}+\mathcal{N}$, while for maximally squeezed light $M_{D}=-\sqrt{\mathcal{N}(\mathcal{N}+1)}$, and for maximally correlated 
classical light $M_{D}=-\mathcal{N}$. The resultant transient coherences are

$$
\begin{aligned}
& \text { Squashed : } \rho_{13} \simeq-\sqrt{\mathcal{N}} t, \\
& \text { Squeezed : } \rho_{13} \simeq-\frac{\sqrt{\mathcal{N}}}{2} t, \\
& \text { Classical : } \rho_{13} \simeq-\frac{\mathcal{N}}{2} t .
\end{aligned}
$$

Again we have assumed that the intensity (or feedback strength) is small, i.e. $\sqrt{\mathcal{N}} \ll 1$. These results clearly show that squashed light de-excitation is a non-classical process. The coherence for squashed light de-excitation (5.20) scales in the same non-classical way as squeezed light (5.21), i.e. $\propto \sqrt{\mathcal{N}} 224$.

For comparison, we can also calculate the two-photon transient coherences for excitation. From Fig. 4 these are given by $\rho_{13} \simeq M_{U} t / 2$. For squeezed and classical light the excitation coherences are exactly the same as the deexcitation coherences in Eqs. (5.21) and (5.22), as one would expect. However, for squashed light $M_{U} \neq M_{D}$ and we obtain

$$
\text { Squashed excitation : } \rho_{13} \simeq \frac{\mathcal{N}}{2} t,
$$

where again this is the weak feedback limit. We see that the two-photon excitation for squashed light (5.23) scales in the same way as classical light (5.22), i.e. $\propto \mathcal{N}$. The transient coherences thus confirm the classical nature of squashed light excitation as indicated by the excited populations in the preceding section.

Thus, in terms of the intensity $\mathcal{N}$, we see a clear scaling difference between the classical (squashed excitation and classical light) and non-classical (squashed de-excitation and squeezed light) processes. For both the steady-state excited populations and the transient two-photon coherences, the non-classical scaling is the square root of the classical scaling.

\section{CONCLUSION}

We have shown in this paper that a unified formalism in terms of effective $N$ and $M$ parameters can be found for squashed, squeezed and classical light. By simply comparing these parameters it appears that squashed light is classical $(M=N)$. Yet there is the contradictory, non-classical fact that it produces photocurrents with noise below the standard quantum limit.

To better understand this unusual non-classical nature of squashed light it is important to look in detail at its interaction with both the two-level, and the cascade threelevel atoms. By doing so, it becomes apparent that there are differences between the upward and downward transitions; $N_{U}=M_{U}$ are quadratically dependent on $\lambda$, the feedback parameter, while $N_{D}=M_{D}$ also have a linear dependence. This difference is not present in either squeezed or classical light, and is a direct consequence of the fact that squashed light is not a free field.

Thus, squashed light does not have a direct correspondence to squeezed light as previous work seemed to indicate [5.:3]. In particular, the observed experimental signature of squeezing (non-classical excited population of a cascade three-level atom [8 10]) is not reproduced. However, the de-excitation of the three-level atom does behave non-classically.

This is most easily seen in the transient two-photon coherence between the highest and lowest levels of an atom prepared in the highest level. The transient coherence scales in the same non-classical way for both squeezing and squashing. In terms of the intensity, $\mathcal{N}=N_{U}$, both the squashed and squeezed coherences are proportional to the square root of the intensity, while classical light has a linear dependence.

To summarize our results, we find that for squashed light, excitation processes are classical, but de-excitation processes are non-classical. This non-classical deexcitation (lowering) is a general feature of squashed light, as evidenced by the line narrowing of the fluorescence of a two-level atom, and the non-classical transient coherence of de-excitation from the upper level of a cascade three-level atom.

[1] D.F. Walls and G.J. Milburn, Quantum Optics (Springer, Berlin,1994).

[2] B.C. Buchler et al, Opt. Lett. 24, 259 (1999).

[3] H.M. Wiseman, J. Opt. B: Quantum Semiclass. Opt. 1, 459 (1999).

[4] The term "squashed" refers to the reduction in fluctuations in one quadrature of the field, with no increase in fluctuations in the other. In squeezed light, by contrast, the fluctuations are "squeezed" into the other quadrature. For a more detailed discussion see Sec. II.B. and Fig. 6.

[5] H.M. Wiseman, Phys. Rev. Lett. 81, 3840 (1998).

[6] C.W. Gardiner, Phys. Rev. Lett. 56, 1917 (1986).

[7] Q.A. Turchette, N.Ph. Georgiades, C.J. Hood, H.J. Kimble, and A.S. Parkins, Phys. Rev. A 58, 4056 (1998).

[8] N.Ph. Georgiades, E.S. Polzik, K.Edamatsu, H.J. Kimble and A.S. Parkins, Phys. Rev. Lett. 75, 3426 (1995).

[9] Z. Ficek and P.D. Drummond, Phys. Rev. A 43, 6247 (1991); ibid. (Part II) 43, 6258 (1991).

[10] Z. Ficek and P.D. Drummond, Physics Today, p.35, September, 1997.

[11] R.E. Slusher et al, Phys. Rev. Lett. 55, 2409 (1985).

[12] B.J. Dalton, Z. Ficek and S. Swain, J. Mod. Opt. 46, 379 (1999).

[13] C.M. Caves and B.L. Schumaker, Phys. Rev. A 31, 3068 (1985).

[14] J.M. Shapiro et al, J. Opt. Soc. Am. B 4, 1604 (1987).

[15] Here the inequality is unity rather than $1 / 16$, as in Ref. [14], because our quadratures, Eq. (2.3), are defined such that $S_{X}=S_{Y}=1$ for the vacuum state. 
[16] R.J. Glauber, Phys. Rev. A 130, 2529 (1963); ibid. 131, 2766 (1963).

[17] E.C.G. Sudarshan, Phys. Rev. Lett. 10, 277 (1963).

[18] C.W. Gardiner, Quantum Noise (Springer, Berlin, 1991).

[19] S. Machida and Y. Yamamoto, Opt. Commun. 57, 290 (1986).

[20] J.J. Stefano, A.R. Subberud and I.J. Williams, Theory and Problems of Feedback and Control Systems 2e (McGraw-Hill, New York, 1990).

[21] Y. Yamamoto, N. Imoto and S. Machida, Phys. Rev. A 33, 3243 (1986).

[22] J.G. Walker and E. Jakeman, Proc. Soc. Photo-Opt. In- strum. Eng. 492, 274 (1995).

[23] H.M. Wiseman, Phys. Rev. A 49, 2133 (1994); Errata ibid. 495159 (1994) and ibid. 50, 4428 (1994).

[24] Another interesting feature is that even for positive feedback, i.e. $\lambda>0$, the in-loop light is not squashed (in fact it has increased noise) but its transient, two-photon coherence for de-excitation still has non-classical scaling. In this case, the relationship between $M_{D}$ and the intensity is now $M_{D}=2 \sqrt{\mathcal{N}}+\mathcal{N}$. Hence, the two-photon coherence $\rho_{13}$ is still proportional to $\sqrt{\mathcal{N}}$; the only difference is the sign. 